Faculty and PostDoc, Medical, Biomedical and Health

Sciences

\title{
Barriers and Motivators of Appropriate Antibiotic Prescription at PHCC in Qatar: Perspective of Physicians and Pharmacists
}

\author{
Sharaf, N.* , AL-Jayyousi, G. ${ }^{* *}$, Radwan, E.*, Shams Eldin, S.* , Hamdani, D., \\ and Habib Sair, A.*
}

Ministry of Public Health*, Department of Public health, College of Health Sciences, Qatar University ${ }^{* *}$

\section{Background}

Antimicrobial resistance (AMR) is a serious public health problem of global concern. The Ministry of Public Health (MOPH) developed the NAP (National Action Plan) to combat AMR in Qatar in collaboration with WHO/EMRO.

Tailoring Antimicrobial Resistance Program is a behavior change methodology that is utilized in this study to tailor behavior change in relation to antimicrobial use. The study explores barriers and motivators of appropriate antibiotic prescription from the physicians' and pharmacists' perspectives at primary healthcare centers in Qatar.

\section{Methodology}

Data were collected from 50 participants across two PHC centers; 30 physicians and 20 pharmacists. Two different interview guides were constructed; one for physicians and one for pharmacists. In-depth, face-to-face, five focus groups were conducted and transcribed verbatim.

Inductive qualitative analysis, involving discovering the themes in the interviews, was followed.

Data were analyzed using constant comparative techniques. Each transcript was coded, and new themes were added to the codebook as they emerged.
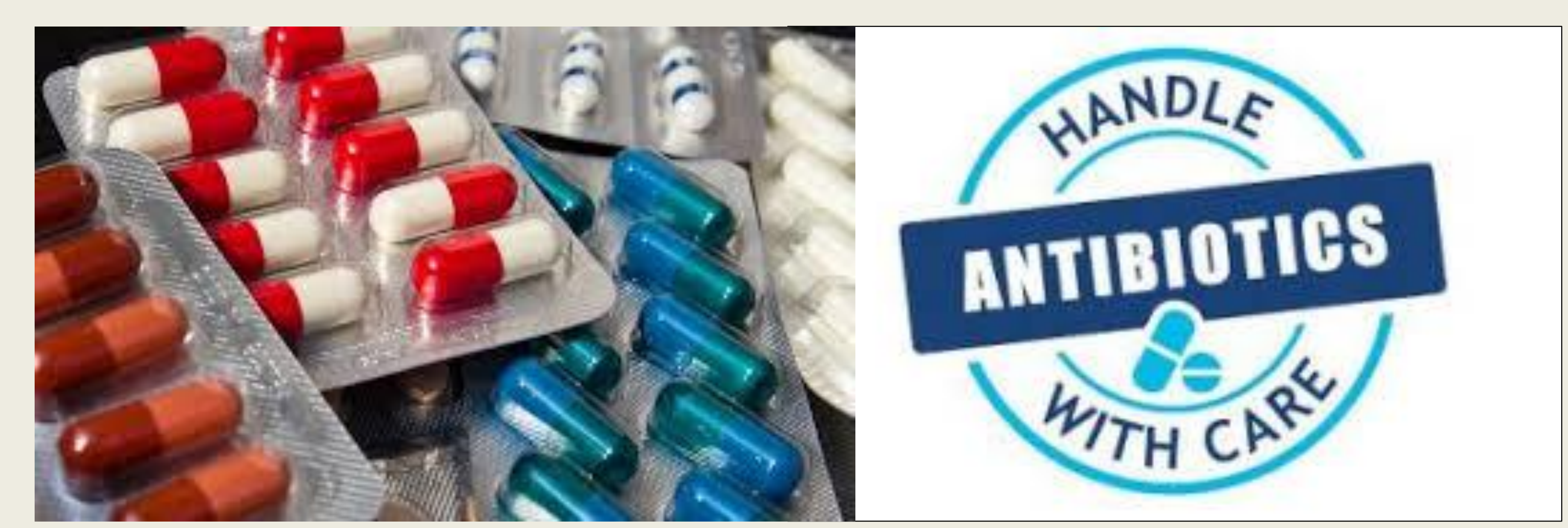

\section{Results}

The analysis revealed that the factors influencing the prescription of antibiotics in PHCC were embedded within the individual, community, and organizational levels of the ecological model.

Participants explained that patients' demographics and practitioners' practices in prescribing $A B$ were among the major barriers.

Key words:

Antibiotic prescription, primary healthcare, focus group discussions. Conflicts of interest:

The authors declare there is no conflict of interest.

\section{Results}

- On the other hand, patient's education and engagement regarding appropriate antibiotic use, physician's ability to make the right decision, and build rapport with the patient to gain trust were among the motivators.

- In addition, auditing AB prescription and the enhancement and utilization of clinical diagnostic tools could play a positive role in changing behavior.

\section{Implications}

The findings would help develop and pilot a behavior change intervention among physicians and pharmacists in the selected PHCCs with the aim of optimizing appropriate antibiotic prescription.

This would support the implementation of the antibiotic stewardship program.

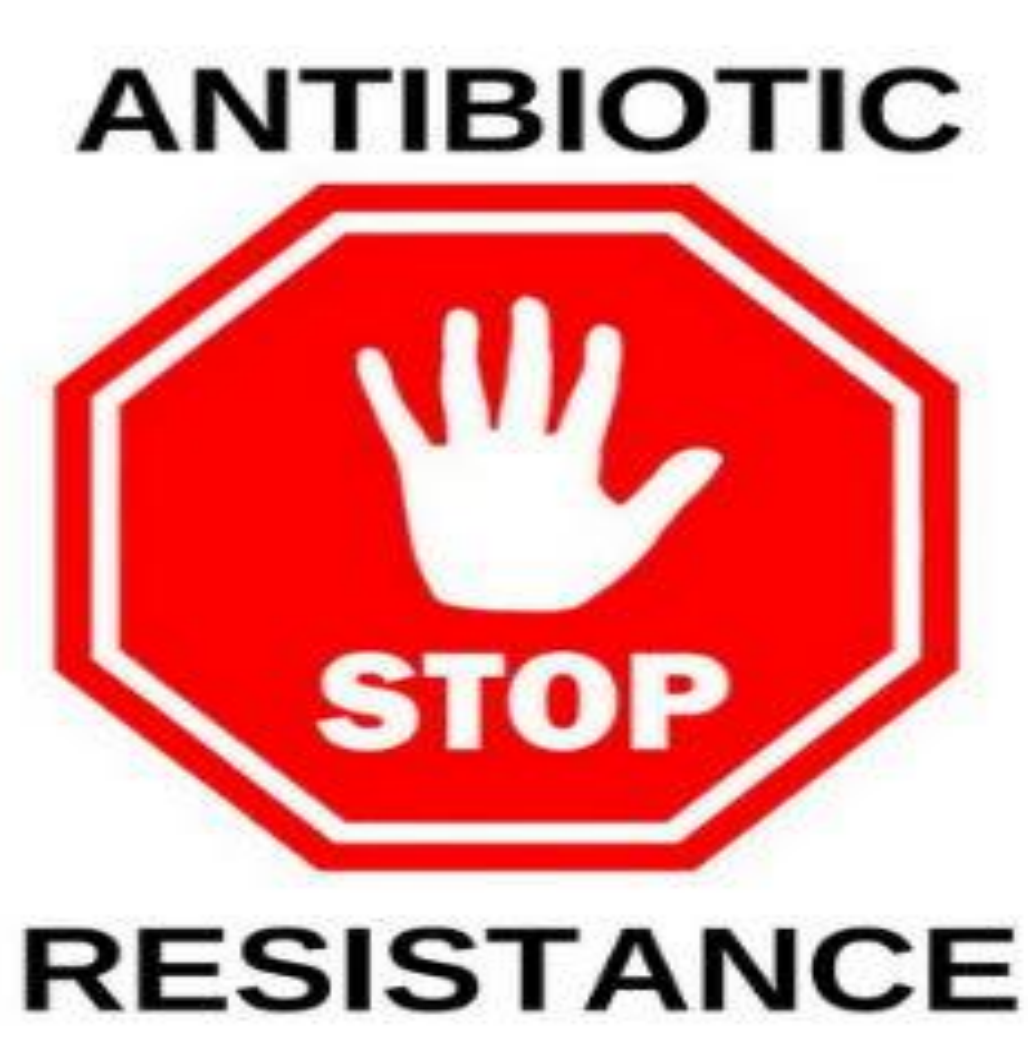

\section{Conclusion}

Effective behavior change interventions should consider multiple factors including individual, community, and organizational factors to optimize appropriate antibiotic prescription, thus decrease the prevalence and burden of antibiotic resistance.

\section{References:}

1. Awad Al, Aboud EA (2015). Knowledge, attitude and practice towards antibiotic use among the public in Kuwait. PLoS One,12;10(2):e0117910. doi: 10.1371/journal.pone.0117910.

2. Butt A, Navasero C, Thomas B, Al Marri S, Al Katheeri H, Al Thani A, et al. Antibiotic prescription patterns for upper respiratory tract infections in the 10 Journal of the Royal Society of Medicine Open 9(9) outpatient Qatari population in the private sector. Int J Infect Dis 2017; 55: 20-23.

3. WHO EMRO j Antimicrobial resistance in the Region $\mathrm{j}$ Antimicrobial resistance j Health topics [Internet]. Emro.who.int. 2017 [cited 13 April 2017]. 